\title{
Darcy-Brinkman Flow Through a Corrugated Channel
}

\author{
Chiu-On Ng - C. Y. Wang
}

Received: 21 October 2009 / Accepted: 31 March 2010 / Published online: 16 April 2010

(C) The Author(s) 2010. This article is published with open access at Springerlink.com

\begin{abstract}
A perturbation analysis is carried out to the second order to give effective equations for Darcy-Brinkman flow through a porous channel with slightly corrugated walls. The flow is either parallel or normal to the corrugations, and the corrugations of the two walls are either in phase or half-period out of phase. The present study is based on the assumptions that the corrugations are periodic sinusoidal waves of small amplitude, and the channel is filled with a sparse porous medium so that the flow can be described by the Darcy-Brinkman model, which approaches the Darcian or Stokes flow limits for small or large permeability of the medium. The Reynolds number is also assumed to be so low that the nonlinear inertia can be ignored. The effects of the corrugations on the flow are examined, quantitatively and qualitatively, as functions of the flow direction, the phase difference, and the wavelength of the corrugations, as well as the permeability of the channel. It is found that the corrugations will have greater effects when it is nearer the Stokes' flow limit than the Darcian flow limit, and when the wavelength is shorter. For the same wavelength and phase difference, cross flow is more affected than longitudinal flow by the corrugations. Opposite effects can result from $180^{\circ}$ out-of-phase corrugations, depending on the flow direction, the wavelength, as well as the permeability.
\end{abstract}

Keywords Stokes flow $\cdot$ Darcy-Brinkman · Corrugated channel

\section{Introduction}

Corrugated walls have long been used in enhancement of heat and mass transfer; see, for example, Bergles (1988). Some recent studies of flow through corrugated channels include,

C.- $\mathrm{O} . \mathrm{Ng}(\otimes)$

Department of Mechanical Engineering, The University of Hong Kong,

Pokfulam Road, Hong Kong, Hong Kong

e-mail: cong@hku.hk

C. Y. Wang

Department of Mathematics, Michigan State University, East Lansing, MI 48824, USA 
among others, Szumbarski and Floryan (2006), Luo et al. (2008), Scholle et al. (2008), Malevich et al. (2008), Khanafer et al. (2009), and Elshafei et al. (2010). One common theme among these studies is to look into the geometrical effects due to wall corrugations on the flow resistance or pressure drop in the channel. The wavy boundary also mimics a rough surface, where the roughness effects are magnified by the small scale of channel width as in micro-fluidics (e.g., Wang and Wang 2007).

In two previous studies by Wang $(1976,1979)$, Stokes' flow between two corrugated plates was examined. It was found that the flow depends not only on the orientation of the corrugations, but also on the phase difference of the corrugations. The phase shift, which changes neither the wetted perimeter nor the cross-sectional area, has different effects on the flow depending on the flow direction. For flow parallel to the corrugations, it is desirable to have the corrugations $180^{\circ}$ out of phase, for which the flow under a given pressure gradient is the maximum and can even be greater than that in the absence of corrugations. In contrast, transverse flow is always decreased by the corrugations. In this orientation, it becomes desirable for the corrugations to be in phase, which gives rise to the smallest flow resistance.

The study is extended here to flow through a layer of porous medium sandwiched between two corrugated plates, which finds applications in filtering equipment, chemical reactors, heat exchangers, and microelectronic cooling devices; see Kaviany (1991), and Nield and Bejan (2006). We are interested to investigate what is the modified effect for flow between corrugations due to the presence of a porous medium.

In this article, we study pressure-driven longitudinal or cross flow through a porous channel that is laterally bounded by corrugated walls. For a sparse porous medium, the DarcyBrinkman model (Brinkman 1947; Kaviany 1991; Ingham and Pop 2002): can be adopted:

$$
\mu_{e} \nabla^{2} \vec{v}-\frac{\mu}{K} \vec{v}=\nabla p
$$

where $\vec{v}$ is the superficial velocity vector, $p$ is the pressure, $\mu_{e}$ is the effective viscosity of the matrix, $\mu$ is the viscosity of the fluid, and $K$ is the permeability. This equation is well accepted for porous media of high porosity such as fiberglass wool. Theoretical support of the Brinkman term for media with low solid fractions can be found from Tam (1969), Lundgren (1972), and Howells (1974). The Darcy-Brinkman equation reduces to the Darcy equation when $K \rightarrow 0$, and to the Stokes equation when $K \rightarrow \infty$.

The flow is confined between two fixed corrugated walls at a distance $2 L$ apart. The corrugation is in the form of a sinusoidal wave of amplitude $\varepsilon L$ and wavenumber $\lambda / L$, where $\varepsilon \ll 1$ so that only slight corrugations are considered. Cartesian coordinates $(x, y, z)$ are defined such that $y=0$ is on the mid-plane of the channel, and $x$ and $z$ are coordinates through the channel in a direction normal and parallel to the corrugations, respectively. We normalize all lengths by $L$, velocities by $G L^{2} / \mu_{e}$, and flow-rate by $G L^{3} / \mu_{e}$, where $G$ is a scale for the pressure gradient. Figure 1 shows a normalized section view of the channel. One should note that, when comparing results, a normalized length in this article is twice that in Wang $(1976,1979)$, as the lengths here are normalized with respect to half the distance between the walls, while the full distance between the walls was used by Wang $(1976,1979)$.

In view of the fact that the maximum or minimum flow will occur at the phase difference $0^{\circ}$ or $180^{\circ}$, we shall only consider the two cases where the upper and lower corrugations are either in phase or half-period out of phase. The upper wavy wall is positioned at

$$
y_{u}(x)=1+\varepsilon \sin \lambda x,
$$

while the lower wavy wall is given by one of these functions

$$
y_{l}^{( \pm)}(x)=-1 \pm \varepsilon \sin \lambda x,
$$




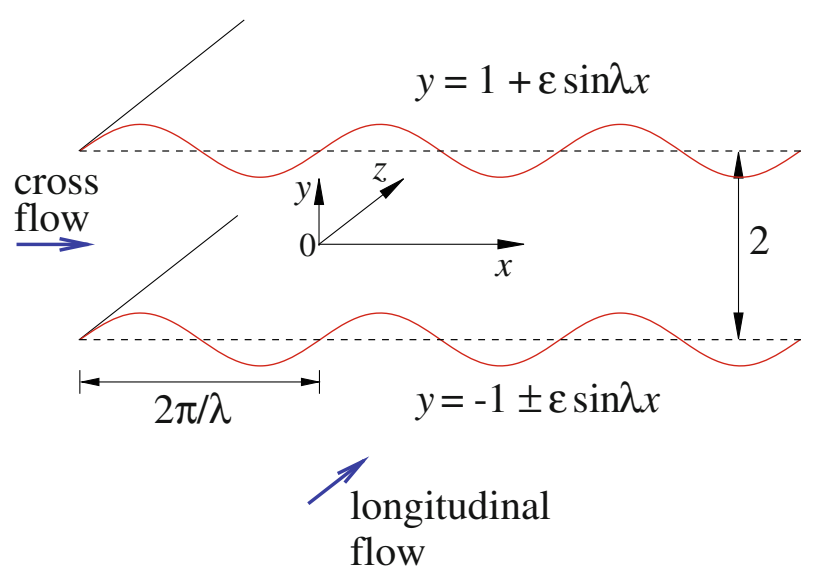

Fig. 1 Normalized section view of longitudinal and cross flows through a corrugated channel. The upper wall is at $y=1+\varepsilon \sin \lambda x$, and the lower wall is at $y=-1 \pm \varepsilon \sin \lambda x$, where $\varepsilon$ and $\lambda$ are the amplitude and wavenumber of the corrugations, respectively, and the plus (minus) sign corresponds to a phase difference of $0^{\circ}\left(180^{\circ}\right)$ between the two walls

where the top (bottom) sign is for the case when the corrugations are in (half-period out of) phase. The small amplitude $\varepsilon \ll 1$ will be used as the perturbation parameter for the problems described below. The dimensionless wavenumber is assumed to be of order unity in general, $\lambda=O(1)$, and yet our model is valid for high wavenumbers as long as $\varepsilon^{2} \lambda \ll 1$. In addition to Wang $(1976,1979)$, similar perturbation analyses for flow in wavy channels have been performed by Chow et al. (1971), and Wang and Wang (2007). All these, however, pertain to pure viscous flow only.

\section{Longitudinal flow}

\subsection{Analysis}

Suppose the flow is driven by a constant pressure gradient $-p_{z}=G$ in the $z$-direction, which is parallel to the corrugations. The flow is purely in the $z$-direction, and the velocity $w(x, y)$ is governed by

$$
\left(\nabla^{2}-k^{2}\right) w=-1
$$

and the no-slip condition at the upper and lower walls $w\left(x, y_{u, l}\right)=0$, where $\nabla \equiv$ $(\partial / \partial x, \partial / \partial y)$, and

$$
k^{2}=\frac{\mu L^{2}}{\mu_{e} K}
$$

is a parameter characterizing the porous medium. The Stokes and Darcian flow limits are given by $k \rightarrow 0$ and $k \gg 1$, respectively.

Let us substitute the following expansion

$$
w=w_{0}+\varepsilon w_{1}+\varepsilon^{2} w_{2}+\cdots,
$$


into Eq.4, and also into the no-slip boundary condition $w=0$ at $y=y_{u}$ and $y=y_{l}^{( \pm)}$, which are Taylor-expanded about the mean wall positions $y=1$ and $y=-1$ as follows:

$$
\begin{aligned}
0= & \left.w\right|_{y=1+\varepsilon \sin \lambda x} \\
= & \left(w_{0}+\varepsilon w_{1}+\varepsilon^{2} w_{2}\right)_{y=1}+\varepsilon \sin \lambda x\left(w_{0 y}+\varepsilon w_{1 y}\right)_{y=1} \\
& +\frac{1}{2} \varepsilon^{2} \sin ^{2} \lambda x\left(w_{0 y y}\right)_{y=1}+O\left(\varepsilon^{3}\right), \\
0= & \left.w\right|_{y=-1 \pm \varepsilon \sin \lambda x} \\
= & \left(w_{0}+\varepsilon w_{1}+\varepsilon^{2} w_{2}\right)_{y=-1} \pm \varepsilon \sin \lambda x\left(w_{0 y}+\varepsilon w_{1 y}\right)_{y=-1} \\
& +\frac{1}{2} \varepsilon^{2} \sin ^{2} \lambda x\left(w_{0 y y}\right)_{y=-1}+O\left(\varepsilon^{3}\right) .
\end{aligned}
$$

On collecting terms of equal powers of $\varepsilon$, we get the perturbation equations as detailed below.

At $O(1)$, the problem is

$$
\left(\nabla^{2}-k^{2}\right) w_{0}=-1
$$

with $w_{0}=0$ at $y=1$ and $y=-1$. The solution, which is independent of $x$, is simply

$$
w_{0}(y)=\frac{1}{k^{2}}\left(1-\frac{\cosh k y}{\cosh k}\right) .
$$

This leading order solution corresponds to the flow in a channel with plane walls.

At $O(\varepsilon)$, the problem is

$$
\left(\nabla^{2}-k^{2}\right) w_{1}=0
$$

with the boundary conditions

$$
\begin{aligned}
w_{1} & =-w_{0 y} \sin \lambda x=\frac{\tanh k}{k} \sin \lambda x & \text { at } y=1, \\
w_{1}^{( \pm)}=\mp w_{0 y} \sin \lambda x & =\mp \frac{\tanh k}{k} \sin \lambda x & \text { at } y=-1 .
\end{aligned}
$$

The solution can be readily found as

$$
w_{1}^{( \pm)}(x, y)=\frac{\tanh k}{k} \sin \lambda x \times\left\{\begin{array}{l}
\frac{\sinh \gamma y}{\sinh \gamma} \\
\frac{\cosh \gamma y}{\cosh \gamma}
\end{array},\right.
$$

where $\gamma^{2}=k^{2}+\lambda^{2}$. This solution gives the first-order perturbation effect of the corrugations on the flow. Its mean over one wavelength is, however, zero, and, therefore, we need to consider the next order to determine the non-zero mean effect.

At $O\left(\varepsilon^{2}\right)$, the problem is

$$
\left(\nabla^{2}-k^{2}\right) w_{2}=0,
$$

with the boundary conditions

$$
\begin{aligned}
w_{2}^{( \pm)} & =\left\{\begin{array}{l}
-w_{1 y} \sin \lambda x-\frac{1}{2} w_{0 y y} \sin ^{2} \lambda x \\
\mp w_{1 y} \sin \lambda x-\frac{1}{2} w_{0 y y} \sin ^{2} \lambda x
\end{array}\right. \\
& =\left\{\begin{array}{l}
\frac{1}{2}(1-\cos 2 \lambda x)\left(\frac{1}{2}-\frac{\gamma}{k} \tanh k \operatorname{coth} \gamma\right) \\
\frac{1}{2}(1-\cos 2 \lambda x)\left(\frac{1}{2}-\frac{\gamma}{k} \tanh k \tanh \gamma\right)
\end{array} \text { at } y=1,-1 .\right.
\end{aligned}
$$


The solution can be expressed as

$$
w_{2}^{( \pm)}(x, y)=g^{( \pm)}(y)-\cos 2 \lambda x \times h^{( \pm)}(y),
$$

where, since our focus is on the non-zero mean, we only need to solve for the non-periodic part of the velocity

$$
g^{( \pm)}(y)=\frac{\cosh k y}{2 \cosh k} \times\left\{\begin{array}{l}
\left(\frac{1}{2}-\frac{\gamma}{k} \tanh k \operatorname{coth} \gamma\right) \\
\left(\frac{1}{2}-\frac{\gamma}{k} \tanh k \tanh \gamma\right)
\end{array}\right.
$$

The flow-rate per unit width of channel is

$$
q(x)=\int_{y_{l}}^{y_{u}} w \mathrm{~d} y
$$

which, upon the substitution of Eqs. (2), (3) and (6) and the Taylor expansion of the integral about the mean wall positions, can be written as

$$
\begin{aligned}
q(x)= & \int_{-1}^{1} w_{0} \mathrm{~d} y+\varepsilon \int_{-1}^{1} w_{1}^{( \pm)} \mathrm{d} y+\varepsilon^{2}\left\{\int_{-1}^{1} w_{2}^{( \pm)} \mathrm{d} y\right. \\
& +\sin \lambda x\left[\left.\left.w_{1}^{( \pm)}\right|_{y=1} \mp w_{1}^{( \pm)}\right|_{y=-1}\right] \\
& \left.+\frac{1}{2} \sin ^{2} \lambda x\left[\left.w_{0}^{\prime}\right|_{y=1}-\left.w_{0}^{\prime}\right|_{y=-1}\right]\right\}+\cdots .
\end{aligned}
$$

On averaging over one wavelength of the corrugations, we get the mean velocity as follows:

$$
\begin{aligned}
\bar{w}^{( \pm)} & =\frac{\lambda}{4 \pi} \int_{0}^{\frac{2 \pi}{\lambda}} q \mathrm{~d} x \\
& =\frac{1}{2} \int_{-1}^{1} w_{0} \mathrm{~d} y+\frac{\varepsilon^{2}}{2}\left(\int_{-1}^{1} g^{( \pm)} \mathrm{d} y+\frac{\tanh k}{2 k}\right)+O\left(\varepsilon^{4}\right) \\
& =\bar{w}_{0}\left(1+\varepsilon^{2} \chi^{( \pm)}+O\left(\varepsilon^{4}\right)\right)
\end{aligned}
$$

where

$$
\bar{w}_{0}=\frac{1}{k^{2}}\left(1-\frac{\tanh k}{k}\right)
$$

is the mean velocity when the walls are perfectly smooth, and

$$
\chi^{( \pm)}(k, \lambda)=\frac{\tanh k}{2 k \bar{w}_{0}} \times\left\{\begin{array}{l}
\left(1-\frac{\gamma}{k} \tanh k \operatorname{coth} \gamma\right) \\
\left(1-\frac{\gamma}{k} \tanh k \tanh \gamma\right)
\end{array}\right.
$$

are functions representing the leading-order perturbation to the mean velocity due to the corrugations; the mean velocity is increased (decreased) when $\chi$ is positive (negative). 
In the Stokes' flow limit (i.e., permeability $k \rightarrow 0$ ), the functions $\chi^{( \pm)}$have the following expressions, which can be checked to agree with that deduced previously by Wang (1976):

$$
\lim _{k \rightarrow 0} \chi^{( \pm)}=\frac{3}{2} \times\left\{\begin{array}{c}
(1-\lambda \operatorname{coth} \lambda) \\
(1-\lambda \tanh \lambda)
\end{array},\right.
$$

which are functions of the wavenumber $\lambda$. Further, if the limits of very long and short wavelengths of corrugations are considered

$$
\lim _{k \rightarrow 0, \lambda \ll 1} \chi^{( \pm)}=\frac{3}{2} \times\left\{\begin{array}{l}
\left(-\lambda^{2} / 3\right) \\
1
\end{array}, \quad \lim _{k \rightarrow 0, \lambda \gg 1} \chi^{( \pm)}=-\frac{3}{2}(\lambda-1)<0 .\right.
$$

For any $\lambda, \chi^{(-)}>\chi^{(+)}$. At the long wave limit, the functions $\chi^{(-)}=1.5$ and $\chi^{(+)}=0$, while at the short wave limit, they are negative tending to the same asymptote.

In the other limit of Darcian flow (i.e., $k \gg 1$ ), the functions $\chi^{( \pm)}$take the following limits for very long and short wavelengths of corrugations:

$$
\begin{aligned}
& \lim _{k \gg 1, \lambda \ll 1} \chi^{( \pm)}=\left\{\begin{array}{l}
-\frac{\lambda^{2}}{4(k-1)}[1+k(\tanh k-\operatorname{coth} k)] \\
\frac{k^{2}}{2(k-1)}\left[1-\left(1+\frac{\lambda^{2}}{2 k^{2}}\right) \tanh ^{2} k\right]
\end{array},\right. \\
& \lim _{k \gg 1, \lambda \gg 1} \chi^{( \pm)}=\frac{k^{2}}{2(k-1)}\left(1-\frac{\gamma}{k}\right)<0 .
\end{aligned}
$$

Again, for any $\lambda, \chi^{(-)}>\chi^{(+)}$, but the difference becomes infinitesimal as the Darcian flow limit is approached.

\subsection{Results and discussion}

Let us look into some more general results. The functions $\chi^{( \pm)}$are plotted against $\lambda$ in Fig. 2 , for $k=0,1$, and 5 . The first case $(k=0)$ corresponds to Stokes' flow, and similar results (noting that the normalized lengths differ by a factor 2) have been presented by Wang (1976). The flow is always decreased when the corrugations are in phase; $\chi^{(+)}$is always negative for any positive $\lambda$ ), but can be increased when the corrugations are out of phase and the wavelength is long enough $\left(\chi^{(-)}\right.$is positive for sufficiently small $\left.\lambda\right)$. Shortening the wavelength of corrugations amounts to increasing the wetted perimeter, and hence will increase the flow resistance. The phase difference becomes immaterial when the wavelength is so short that the wavenumber $\lambda>3$ (the $\chi^{(+)}$and $\chi^{(-)}$curves tend to the same asymptote, given in Eq. 25 , as $\lambda$ increases).

The presence of porous materials in the channel will in general diminish the effect of the corrugations on the flow. As $k$ increases or the channel becomes less permeable, the functions $\chi^{( \pm)}$, whether positive or negative, will decrease in magnitude for given $\lambda$. Also, the phase difference becomes unimportant when the permeability is so low that $k>5$ is approaching the Darcian flow limit.

The effect of $k$ on $\chi^{( \pm)}$is further shown in Fig. 3, where $\lambda=1$. Indeed, the corrugations will have the maximum relative effects on the flow when the channel is perfectly permeable, i.e., filled with fluid only. The decrease in magnitude of the two functions is sharp in the initial range $0<k<2$, and is much milder thereafter. The in-phase function $\chi^{(+)}$decreases in magnitude monotonically as $k$ increases. The out-of-phase function $\chi^{(-)}$, however, drops to a negative maximum before it joins $\chi^{(+)}$to tend to zero as $k$ increases. The corrugations will continuously lose their influence on the flow as the permeability decreases. 

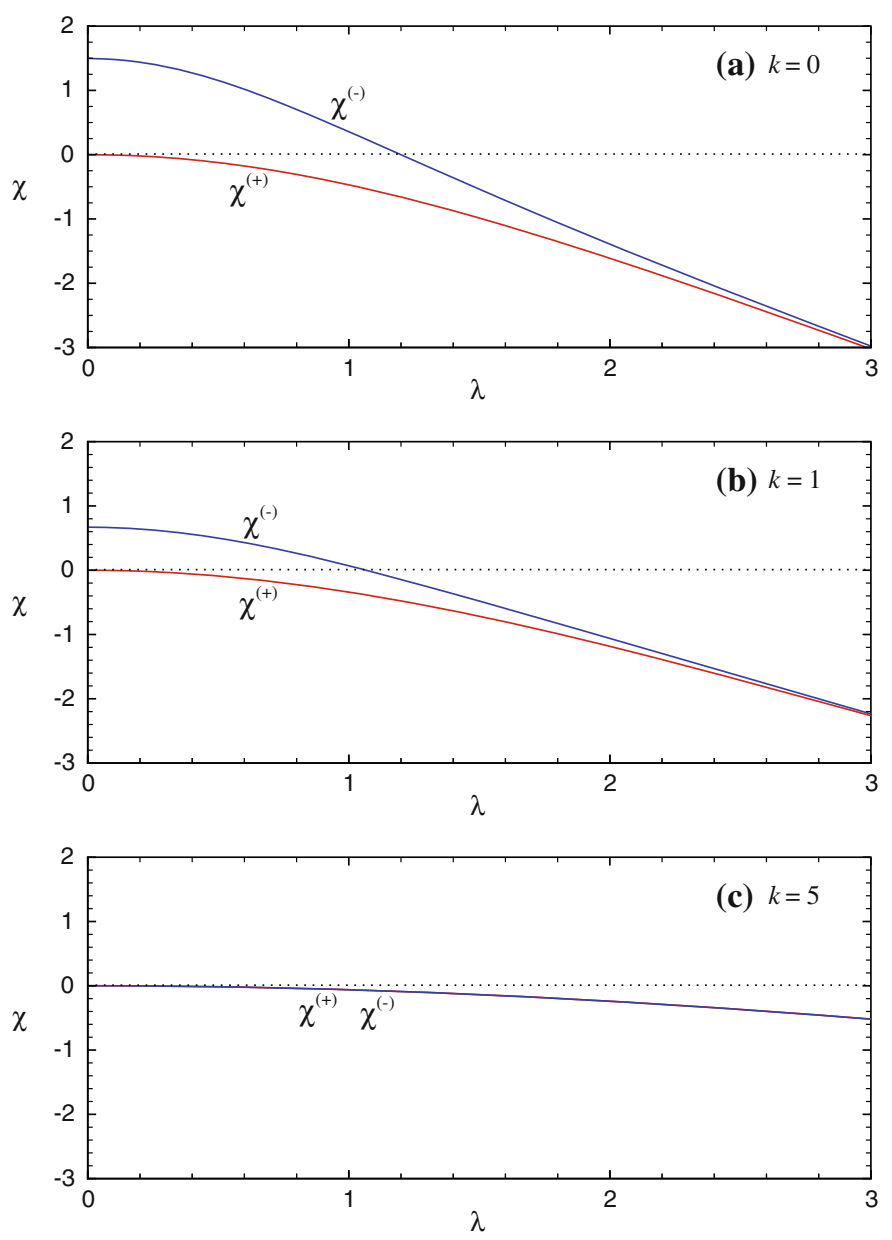

Fig. 2 The effect of corrugations on longitudinal flow: $\chi^{( \pm)}$, which is given in Eq. 23, as functions of the wavenumber $\lambda$, for the medium parameter $\mathbf{a} k=0$ (Stokes flow limit), $\mathbf{b} k=1, \mathbf{c} k=5$. The plus (minus) sign is for in-phase $\left(180^{\circ}\right.$ out-of-phase) corrugations. In c, the two curves are practically indistinguishable. The dotted lines stand for $\chi=0$. Note that positive (negative) $\chi$ means that the flow rate is increased (decreased) by the corrugations

The wavenumber, denoted by $\lambda_{0}$, at which the out-of-phase function $\chi^{(-)}$crosses zero, is shown in Fig. 4, as a function of $k$. The function $\chi^{(-)}$is positive over the range $0<\lambda<\lambda_{0}$. The range is the widest when $k=0$ and $\lambda_{0}=1.1997$, a value noted previously by Wang (1976). The threshold wavenumber $\lambda_{0}$ drops rather quickly as $k$ increases from 0 to 4 , and becomes virtually zero when $k>5$. This means that the enhancement of flow due to out-ofphase corrugations will not happen in the Darcian flow regime.

\section{Cross flow}

Suppose the mean flow is now purely in the $x$-direction, i.e., normal to the corrugations, and the flow-rate is known to be $Q$. The mean $x$-pressure gradient, which is scaled by 
$G=\mu_{e} Q / 2 L^{3}$, will be determined later. The cross flow field is two-dimensional, and has periodic as well as non-periodic components.

\subsection{Analysis}

Reverting to the normalized variables, we may introduce a streamfunction $\psi(x, y)$ such that the velocity components $(u, v)=\left(\psi_{y},-\psi_{x}\right)$. The Darcy-Brinkman Equation 1$)$ becomes

$$
\begin{aligned}
& \left(\nabla^{2}-k^{2}\right) \psi_{y}=p_{x}, \\
& \left(\nabla^{2}-k^{2}\right) \psi_{x}=-p_{y},
\end{aligned}
$$

where $\nabla \equiv(\partial / \partial x, \partial / \partial y)$, and $k$ is given by Eq. 5 . Eliminating $p$ from these equations gives

$$
\nabla^{2}\left(\nabla^{2}-k^{2}\right) \psi=0
$$

which implies $\nabla^{2} \psi=0$ or $\left(\nabla^{2}-k^{2}\right) \psi=0$. The kinematic and no-slip boundary conditions are

$$
\begin{aligned}
& \psi=1, \quad \psi_{n}=0 \quad \text { at } y=y_{u}, \\
& \psi=-1, \quad \psi_{n}=0 \quad \text { at } y=y_{l}^{( \pm)},
\end{aligned}
$$

where $y_{u}(x)$ and $y_{l}^{( \pm)}(x)$ are the upper and lower wavy wall positions, as given by Eqs. (2) and (3), respectively. The normalized rate of flow through unit width of the channel is equal to 2 . The vanishing of the normal derivative of $\psi$ (i.e., $\psi_{n}=\psi_{x} n_{x}+\psi_{y} n_{y}=0$ where $n_{x}$ and $n_{y}$ are the $x$ - and $y$-components of the unit normal) at the boundaries can be expressed as

$$
\begin{array}{ll}
-(\varepsilon \lambda \cos \lambda x) \psi_{x}+\psi_{y}=0 & \text { at } y=y_{u}, \\
\mp(\varepsilon \lambda \cos \lambda x) \psi_{x}+\psi_{y}=0 & \text { at } y=y_{l}^{( \pm)} .
\end{array}
$$

Let us substitute the following expansion

$$
\psi=\psi_{0}+\varepsilon \psi_{1}+\varepsilon^{2} \psi_{2}+\cdots
$$

into Eqs.30-32. The two boundary conditions are Taylor-expanded about the mean wall positions $y=1$ and $y=-1$ to become:

$$
\begin{aligned}
1= & \psi_{0}+\varepsilon \psi_{1}+\varepsilon^{2} \psi_{2}+\varepsilon \sin \lambda x\left(\psi_{0 y}+\varepsilon \psi_{1 y}\right) \\
& +\frac{1}{2} \varepsilon^{2} \sin ^{2} \lambda x\left(\psi_{0 y y}\right)+O\left(\varepsilon^{3}\right) \quad \text { at } y=1, \\
0= & -(\varepsilon \lambda \cos \lambda x)\left(\phi_{0 x}+\varepsilon \phi_{1 x}\right)+\psi_{0 y}+\varepsilon \psi_{1 y}+\varepsilon^{2} \psi_{2 y} \\
& +\varepsilon \sin \lambda x\left[-(\varepsilon \lambda \cos \lambda x) \phi_{0 x y}+\psi_{0 y y}+\varepsilon \psi_{1 y y}\right] \\
& +\frac{1}{2} \varepsilon^{2} \sin ^{2} \lambda x\left(\phi_{0 y y y}\right)+O\left(\varepsilon^{3}\right) \quad \text { at } y=1, \\
-1= & \psi_{0}+\varepsilon \psi_{1}+\varepsilon^{2} \psi_{2} \pm \varepsilon \sin \lambda x\left(\psi_{0 y}+\varepsilon \psi_{1 y}\right) \\
& +\frac{1}{2} \varepsilon^{2} \sin ^{2} \lambda x\left(\psi_{0 y y}\right)+O\left(\varepsilon^{3}\right) \quad \text { at } y=-1, \\
0= & \mp(\varepsilon \lambda \cos \lambda x)\left(\phi_{0 x}+\varepsilon \phi_{1 x}\right)+\psi_{0 y}+\varepsilon \psi_{1 y}+\varepsilon^{2} \psi_{2 y} \\
& \pm \varepsilon \sin \lambda x\left[\mp(\varepsilon \lambda \cos \lambda x) \phi_{0 x y}+\psi_{0 y y}+\varepsilon \psi_{1 y y}\right] \\
& +\frac{1}{2} \varepsilon^{2} \sin ^{2} \lambda x\left(\phi_{0 y y y}\right)+O\left(\varepsilon^{3}\right) \quad \text { at } y=-1 .
\end{aligned}
$$

On collecting terms of equal powers of $\varepsilon$, we get the perturbation equations as detailed below. 


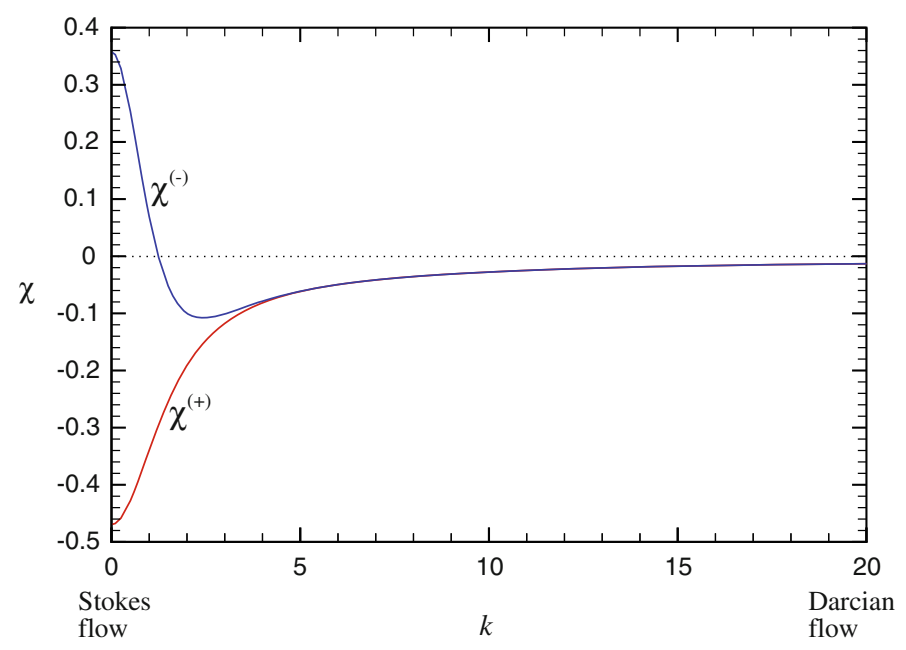

Fig. 3 The effect of corrugations on longitudinal flow: $\chi^{( \pm)}$as functions of $k$, for $\lambda=1$. The plus (minus) sign is for in-phase $\left(180^{\circ}\right.$ out-of-phase) corrugations. The dotted line stands for $\chi=0$

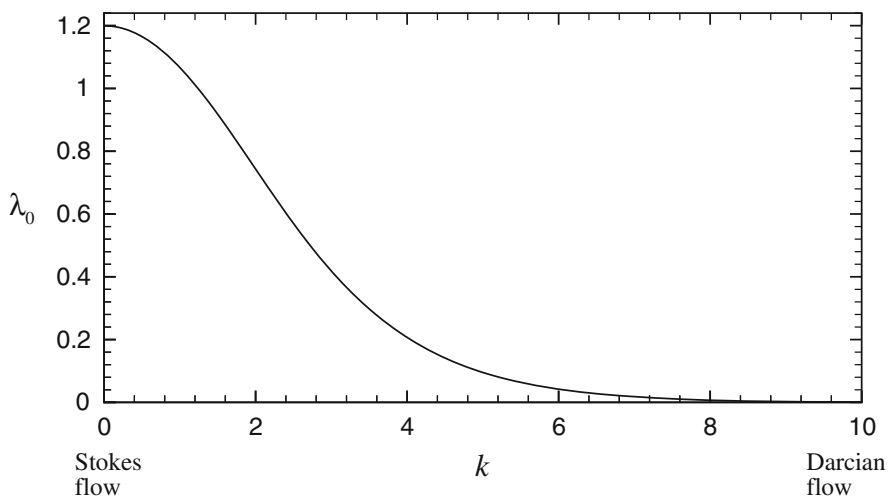

Fig. 4 The wavenumber $\lambda_{0}$, below which $\chi^{(-)}>0$, as a function of $k$. $\lambda_{0}=1.1997$ when $k=0$

The $O(1)$ problem is

$$
\nabla^{2}\left(\nabla^{2}-k^{2}\right) \psi_{0}=0
$$

with the boundary conditions

$$
\begin{aligned}
& \psi_{0}=1, \quad \psi_{0 y}=0 \quad \text { at } y=1, \\
& \psi_{0}=-1, \quad \psi_{0 y}=0 \quad \text { at } y=-1 .
\end{aligned}
$$

Obviously, $\psi_{0}$ is a pure odd function of $y$. The solution is

$$
\psi_{0}(y)=c_{1} y+c_{2} \sinh k y,
$$

where

$$
c_{2}=-\frac{c_{1}}{k \cosh k}=\frac{1}{\sinh k-k \cosh k} .
$$


The $O(\varepsilon)$ problem is

$$
\nabla^{2}\left(\nabla^{2}-k^{2}\right) \psi_{1}=0
$$

with the boundary conditions

$$
\left.\begin{array}{l}
\left.\begin{array}{l}
\psi_{1}=0 \\
\psi_{1 y}=-c_{2} k^{2} \sinh k \sin \lambda x
\end{array}\right\} \quad \text { at } y=1, \\
\psi_{1}=0 \\
\psi_{1 y}^{( \pm)}= \pm c_{2} k^{2} \sinh k \sin \lambda x
\end{array}\right\} \text { at } y=-1 .
$$

The solution is

$$
\psi_{1}^{( \pm)}(x, y)=c_{2} k^{2} \sinh k \sin \lambda x \times\left\{\begin{array}{l}
c_{3} \cosh \lambda y+c_{4} \cosh \gamma y \\
c_{5} \sinh \lambda y+c_{6} \sinh \gamma y
\end{array}\right.
$$

where $\gamma^{2}=k^{2}+\lambda^{2}$, and

$$
\begin{aligned}
& c_{4}=-c_{3} \frac{\cosh \lambda}{\cosh \gamma}=\frac{\cosh \lambda}{\lambda \sinh \lambda \cosh \gamma-\gamma \sinh \gamma \cosh \lambda}, \\
& c_{6}=-c_{5} \frac{\sinh \lambda}{\sinh \gamma}=\frac{\sinh \lambda}{\lambda \sinh \gamma \cosh \lambda-\gamma \sinh \lambda \cosh \gamma} .
\end{aligned}
$$

The $O\left(\varepsilon^{2}\right)$ problem is

$$
\nabla^{2}\left(\nabla^{2}-k^{2}\right) \psi_{2}=0
$$

with the boundary conditions

$$
\left.\begin{array}{l}
\left.\begin{array}{l}
\psi_{2}=\frac{1}{4} c_{2} k^{2} \sinh k(1-\cos 2 \lambda x) \\
\psi_{2 y}^{( \pm)}=-\frac{1}{2} c_{2} c_{7}^{( \pm)} k^{2} \sinh k(1-\cos 2 \lambda x)
\end{array}\right\} \quad \text { at } y=1, \\
\psi_{2}=-\frac{1}{4} c_{2} k^{2} \sinh k(1-\cos 2 \lambda x) \\
\psi_{2 y}^{( \pm)}=-\frac{1}{2} c_{2} c_{7}^{( \pm)} k^{2} \sinh k(1-\cos 2 \lambda x)
\end{array}\right\} \quad \text { at } y=-1,
$$

where

$$
c_{7}^{( \pm)}=\frac{k}{2 \tanh k}+\left\{\begin{array}{l}
k^{2} /(\lambda \tanh \lambda-\gamma \tanh \gamma) \\
k^{2} /(\lambda \operatorname{coth} \lambda-\gamma \operatorname{coth} \gamma)
\end{array} .\right.
$$

Again, we only need to solve for the non-periodic part of $\psi_{2}$ given by the average over one wavelength of the corrugations

$$
\bar{\psi}_{2}(y)=\frac{\lambda}{2 \pi} \int_{0}^{2 \pi / \lambda} \psi_{2} \mathrm{~d} x
$$

The solution is odd in $y$, and is found to be

$$
\bar{\psi}_{2}^{( \pm)}(y)=c_{8}^{( \pm)} y+c_{9}^{( \pm)} \sinh k y
$$


where

$$
\begin{aligned}
& c_{8}^{( \pm)}=-\frac{k^{2} \sinh k\left(2 c_{7}^{( \pm)} \sinh k+k \cosh k\right)}{4(\sinh k-k \cosh k)^{2}}, \\
& c_{9}^{( \pm)}=\frac{k^{2} \sinh k\left(1+2 c_{7}^{( \pm)}\right)}{4(\sinh k-k \cosh k)^{2}} .
\end{aligned}
$$

Taking the average of Eq. 28 over one wavelength of the corrugations, we get the mean $x$-pressure gradient as given by

$$
\begin{aligned}
\bar{p}_{x}^{( \pm)} & =\left(\frac{\mathrm{d}^{2}}{\mathrm{~d} y^{2}}-k^{2}\right) \bar{\psi}_{y}^{( \pm)} \\
& =\left(\frac{\mathrm{d}^{2}}{\mathrm{~d} y^{2}}-k^{2}\right)\left(\psi_{0 y}+\varepsilon^{2} \bar{\psi}_{2 y}^{( \pm)}+O\left(\varepsilon^{4}\right)\right) \\
& =-k^{2} c_{1}\left(1+\varepsilon^{2} \zeta^{( \pm)}+O\left(\varepsilon^{4}\right)\right),
\end{aligned}
$$

where $p_{x}=-k^{2} c_{1}$ is the pressure gradient if the walls were smooth, and

$$
\zeta^{( \pm)}(k, \lambda)=\frac{c_{8}^{( \pm)}}{c_{1}}=\frac{k \tanh k\left(2 c_{7}^{( \pm)} \sinh k+k \cosh k\right)}{4(\sinh k-k \cosh k)}
$$

are functions representing the effects of the corrugations on the mean pressure gradient required to drive the given rate of flow transverse to the corrugations. In terms of dimensional pressure gradient and flow rate, Eq. 59 can be inverted to become an equation for flow rate per unit pressure gradient

$$
\frac{Q^{( \pm)}}{2 L^{3}\left(-\bar{p}_{x}\right) /\left(k^{2} c_{1} \mu_{e}\right)}=1-\varepsilon^{2} \zeta^{( \pm)}+O\left(\varepsilon^{4}\right)
$$

Hence, for a given mean pressure drop, the flow is increased (decreased) by the corrugations when $\zeta$ is negative (positive).

In the limiting case of Stokes flow (i.e., $k \rightarrow 0$ ), the functions $\zeta^{( \pm)}$have the following expressions, which can be checked to agree with that previously obtained by Wang (1979):

$$
\lim _{k \rightarrow 0} \zeta^{( \pm)}=-\frac{3}{2}+3 \lambda /\left\{\begin{array}{l}
\left(\tanh \lambda+\lambda \operatorname{sech}^{2} \lambda\right) \\
\left(\operatorname{coth} \lambda-\lambda \operatorname{csch}^{2} \lambda\right)
\end{array} .\right.
$$

Further, if the limits of very long and short wavelengths of corrugations are considered

$$
\lim _{k \rightarrow 0, \lambda \ll 1} \zeta^{( \pm)}=\left\{\begin{array}{l}
\lambda^{2} \\
3
\end{array}, \quad \lim _{k \rightarrow 0, \lambda \gg 1} \zeta^{( \pm)}=-\frac{3}{2}+3 \lambda>0 .\right.
$$

For any $\lambda, \zeta^{(-)}>\zeta^{(+)}>0$. At the long wave limit, the functions $\zeta^{(-)}=3$ and $\zeta^{(+)}=0$, while at the short wave limit, they tend to the same asymptote increasing linearly with $\lambda$.

In the other limit of Darcian flow (i.e., $k \gg 1$ ), the functions $\zeta^{( \pm)}$take the following limits for very long and short wavelengths of corrugations:

$$
\lim _{k \gg 1, \lambda \ll 1} \zeta^{( \pm)}=\left\{\begin{array}{l}
\frac{\lambda^{2}}{4}\left(\frac{2 k-1}{k-1}\right) \\
\frac{k^{2}}{2(k-1)^{2}}
\end{array}, \quad \lim _{k \gg 1, \lambda \gg 1} \zeta^{( \pm)}=\frac{k^{2}}{2(k-1)}\left(\frac{k}{\gamma-\lambda}-1\right)>0 .\right.
$$

Again, for any $\lambda, \zeta^{(-)}>\zeta^{(+)}>0$. Unlike the parallel flow, the difference between the two functions at small $\lambda$ remains finite as the Darcian flow limit is approached. 

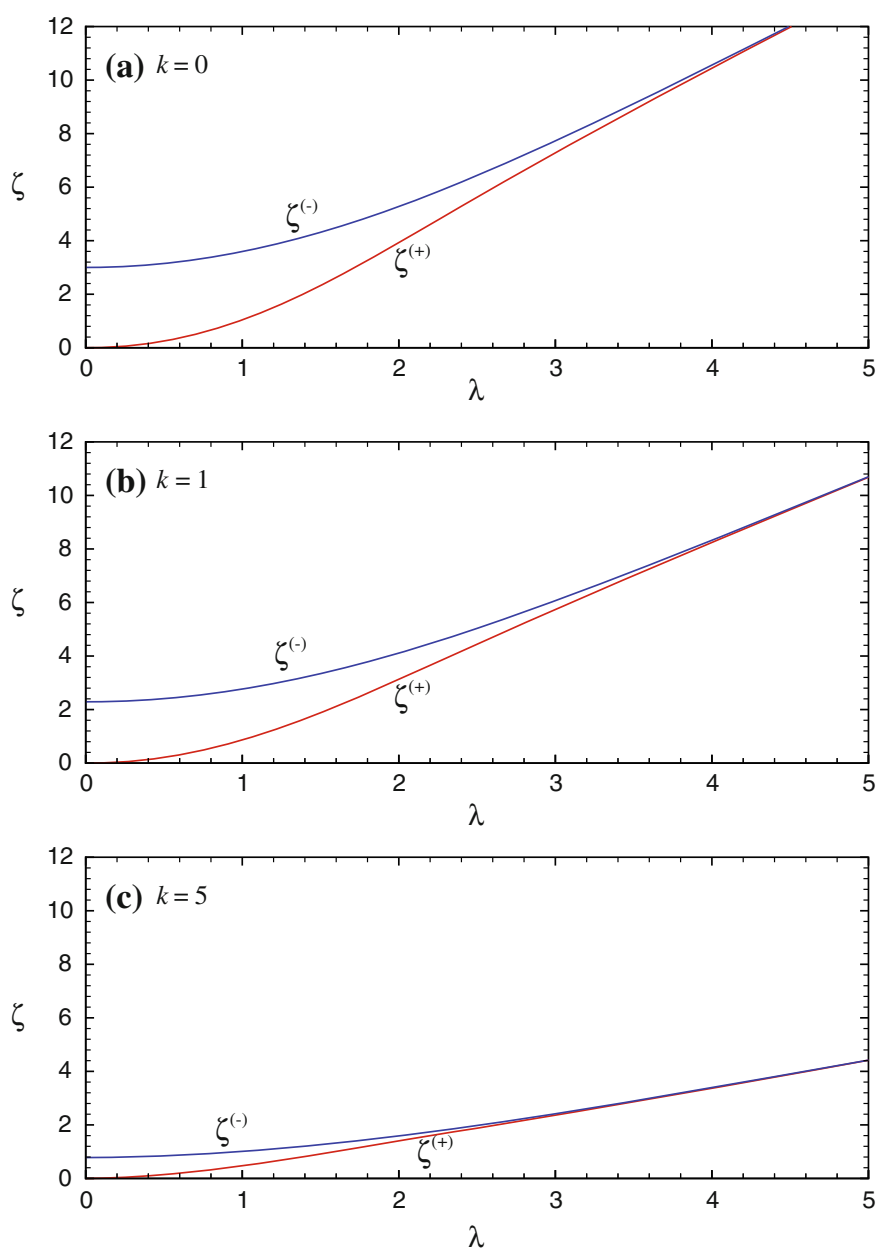

Fig. 5 The effect of corrugations on cross flow: $\zeta^{( \pm)}$, which is given in Eq. 60, as functions of the wavenumber $\lambda$, for the medium parameter $\mathbf{a} k=0$ (Stokes flow limit), $\mathbf{b} k=1, \mathbf{c} k=5$. The plus (minus) sign is for in-phase $\left(180^{\circ}\right.$ out-of-phase) corrugations. Note that larger $\zeta$ means larger flow resistance

\subsection{Results and discussion}

Let us here look into some more general results. The functions $\zeta^{( \pm)}$are plotted against $\lambda$ in Fig. 5, for $k=0,1$, and 5. The first case $(k=0)$ corresponds to Stokes' flow, and similar results (noting that the normalized lengths differ by a factor 2) have been presented by Wang (1979). Cross flow is always decreased by the corrugations irrespective of the phase difference. For given $k$ and $\lambda$, the flow resistance is the smallest when the corrugations are in phase, and the largest when $180^{\circ}$ out of phase. Again, the flow resistance increases when the wavelength of corrugations decreases, and the phase difference becomes immaterial when the wavenumber $\lambda>5$.

On comparing Figs. 2 and 5, one can readily see that the corrugations will in general have greater effects on cross flow than on longitudinal flow; the former may experience several times larger resistance than the latter, depending on $k$ and $\lambda$. 


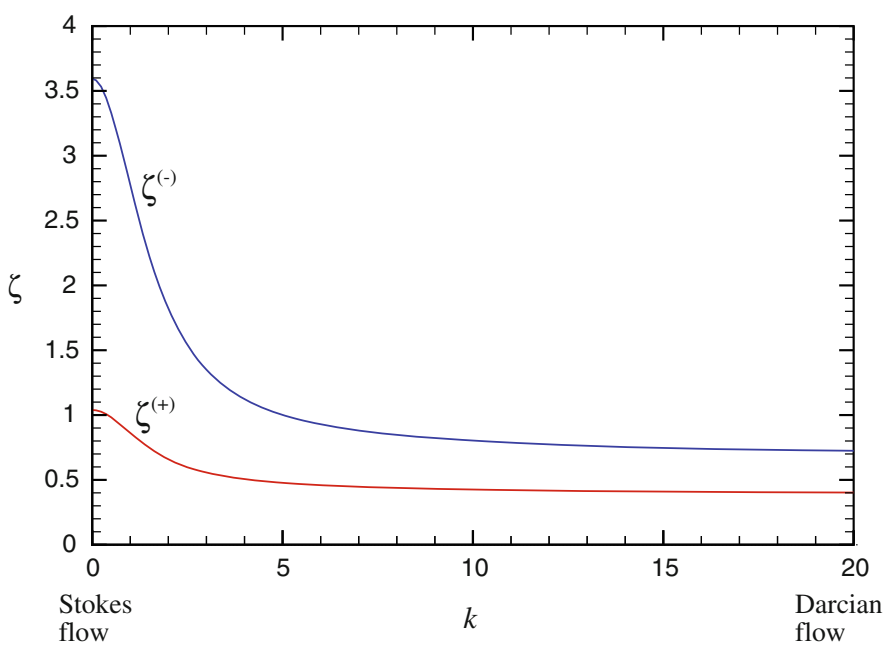

Fig. 6 The effect of corrugations on cross flow: $\zeta^{( \pm)}$as functions of $k$, for $\lambda=1$. The plus (minus) sign is for in-phase $\left(180^{\circ}\right.$ out-of-phase) corrugations

The effect of $k$ on $\zeta^{( \pm)}$is further shown in Fig. 6, where $\lambda=1$. As in the longitudinal case, the presence of porous materials in the channel will diminish the effect of the corrugations on the flow. Also, the corrugations will have the maximum relative effects on the flow in the Stokes flow limit. The decrease in magnitude of the two functions $\zeta^{( \pm)}$is sharp in the early range $0<k<2$, and is much milder thereafter. As mentioned above, the corrugations will continue to have finite effect, although diminished, on the cross flow as the Darcian flow limit is approached. The effect is also dependent on the phase difference for small $\lambda$.

\section{Concluding remarks}

This is the first article on the effect of wavy boundaries on the flow in a porous medium. We have deduced analytical results for the mean longitudinal and cross Darcy-Brinkman flows through a porous channel bounded by slightly corrugated walls. These results are important in micro-fluidic applications where the flow is much affected by wall roughness.

The effects of the corrugations on the flows are represented by the second order terms which are functions of the phase difference, the wavenumber of the corrugations, and a parameter characterizing the permeability of the channel. In summary, the corrugations will tend to lose their influence on the flow as as the Darcian flow limit is approached with increasing permeability; see Figs. 2, 3, 5 and 6. On the other hand, the decreasing effects of the corrugations on the flow will increase when the wavelength decreases, corresponding to increasing wetted perimeter per unit span of channel. For the same wavelength and phase difference, cross flow is in general more affected than longitudinal flow by the corrugations. The former may experience several times larger resistance than the latter, depending on the permeability and wavelength. Under the conditions that the corrugations are $180^{\circ}$ out of phase, and their wavenumber is smaller $\lambda_{0}$ shown in Fig. 4 , corrugations may indeed enhance the rate of longitudinal flow. The phase difference becomes, however, unimportant for sufficiently large wavenumber, and particularly in the Darcian flow limit for longitudinal flow, as shown in Fig. 2c. If for any reason, due to strengthening or increased surface area, the bounding plates 
of a channel are corrugated, then we find it is better to align the striations with the pressure gradient and make the waves $180^{\circ}$ off phase, to maximize the flow.

This study is limited to corrugations of small amplitude. If the amplitudes are large, then the same governing equations hold, but the boundary conditions are difficult to handle analytically. In that case, numerical finite differences (e.g., Zhou et al. 2002) or boundary integrals (e.g., Pozrikidis 1987) may be used.

Acknowledgements The study was initiated by the second author when he was a William Mong Visiting Research Fellow associating with the first author in May, 2008. The financial support by the William M.W. Mong Engineering Research Fund of the University of Hong Kong is gratefully acknowledged. The study was also partly supported by the Research Grants Council of the Hong Kong Special Administrative Region, China, through Project No. HKU 715609E.

Open Access This article is distributed under the terms of the Creative Commons Attribution Noncommercial License which permits any noncommercial use, distribution, and reproduction in any medium, provided the original author(s) and source are credited.

\section{References}

Bergles, A.E.: Some perspectives on enhanced heat-transfer-2nd generation heat-transfer technology. J. Heat Transf. 110, 1082-1096 (1988)

Brinkman, H.C.: A calculation of the viscous force exerted by a flowing fluid in a dense swarm of particles. Appl. Sci. Res. A1, 27-34 (1947)

Chow, J.C.F., Soda, K., Dean, C.: On laminar flow in wavy channels. Proc. 12th Midwestern Mech. Conf. 6, 247-253 (1971)

Elshafei, E.A.M., Awad, M.M., El-Negiry, E., Ali, A.G.: Heat transfer and pressure drop in corrugated channels. Energy 35, 101-110 (2010)

Howells, I.D.: Drag due to the motion of a Newtonian fluid through a sparse random array of small fixed rigid objects. J. Fluid Mech. 64, 449-475 (1974)

Ingham, D.B., Pop, I.: Transport in Porous Media. Pergamon, Oxford (2002)

Kaviany, M.: Principles of Heat Transfer in Porous Media. Springer, New York (1991)

Khanafer, K., Al-Azmi, B., Marafie, A., Pop, I.: Non-Darcian effects on natural convection heat transfer in a wavy porous enclosure. Int. J. Heat Mass Transf. 52, 1887-1896 (2009)

Lundgren, T.S.: Slow flow through stationary random beds and suspensions of spheres. J. Fluid Mech. 51, 273-299 (1972)

Luo, H., Blyth, M.G., Pozrikidis, C.: Two-layer flow in a corrugated channel. J. Eng. Math. 60, 127-147 (2008)

Malevich, A.E., Mityushev, V.V., Adler, P.M.: Couette flow in channels with wavy walls. Acta Mech. 197, 247-283 (2008)

Nield, D.A., Bejan, A.: Convection in Porous Media. 3rd edn. Springer, New York (2006)

Pozrikidis, C.: Creeping flow in two-dimensional channels. J. Fluid Mech. 180, 495-514 (1987)

Scholle, M., Haas, A., Aksel, N., Wilson, M.C.T., Thompson, H.M., Gaskell, P.H.: Competing geometric and inertial effects on local flow structure in thick gravity-driven fluid films. Phys. Fluids 20, 123101 (2008)

Szumbarski, J., Floryan, J.M.: Transient disturbance growth in a corrugated channel. J. Fluid Mech. 568, 243-272 (2006)

Tam, C.K.W.: The drag on a cloud of spherical particles in low Reynolds number flow. J. Fluid Mech. 38, 537-546 (1969)

Wang, C.Y.: Parallel flow between corrugated plates. J. Eng. Mech. 102, 1088-1090 (1976)

Wang, C.Y.: On Stokes flow between corrugated plates. J. Appl. Mech. 46, 462-464 (1979)

Wang, H., Wang, Y.: Flow in microchannels with rough walls: flow pattern and pressure drop. J. Micromech. Microeng. 17, 586-596 (2007)

Zhou, H., Khayat, R.E., Martinuzzi, R.J., Straatman, A.G.: On the validity of the perturbation approach for the flow inside weakly modulated channels. Int. J. Numer. Methods Fluids 39, 1139-1159 (2002) 